\title{
TIEMPO, JUEGO Y CRONOLOGÍA ESCOLAR INDÍGENA. ¿INTERCULTURALIDAD? ${ }^{1}$
}

\author{
TIME, PLAY AND INDIGENOUS SCHOLAR CHRONOLOGY, \\ INTERCULTURALITY?
}

Víctor Alonso Molina Bedoya ${ }^{2}$

Resumen

\begin{abstract}
En este artículo se expone la tensión entre los tiempos de la producción y los del juego/ocio en una comunidad indígena de Colombia. La investigación tuvo como propósito indagar por las relaciones entre el ocio, el desarrollo sostenible y el tiempo en la cosmovisión indígena, lo mismo que los tiempos según el cronosistema escolar. El enfoque fue cualitativo, de método etnográfico. La recolección de la información se hizo a partir de diarios de campo, registros fotográficos y entrevistas semiestructuradas. Se reconoce a la escuela como una institución que establece y delimita los tiempos acorde a un cronosistema que regula los lapsos del trabajo/estudio y del descanso, además de alejar a los pequeños de los ciclos naturales e incorporarlos en la dimensión abstracta del tiempo.
\end{abstract}

Palabras claves: juego, tiempo, cronosistema escolar.

Abstract

The paper exposes the stress between times of production and those of play-leisure in the indigenous community in Colombia. The purpose of this research was to inquire among leisure, sustainable development and time in indigenous worldview and times according to scholar chronosystem. The ethnographical method had a qualitative focus. Data was gathered from campus diaries, photographic registry and semi-structured interviews. The school is recognized as an institution witch establish and delimit times in accord to a cronosystem that regulates those lapses between studding and resting time, besides talks the little natural cycles and incorporate them in the abstract dimension of time.

Keywords: play, time, scholar chromo-system.

Fecha de recepción: 15 de mayo de 2015

Fecha de aprobación: 8 de septiembre de 2015

Para citar este artículo:

Molina, V.A. (2015). Tiempo, juego y cronología escolar indígena. ¿Interculturalidad? Lúdica Pedagógica, (22), 11-19.

1 Artículo asociado a la investigación: Cosmovisión de la unidad: existencia equilibrada, juego-producción y educación en la comunidad indígena Nasa de Caldono, Colombia.

2 Doctor. Profesor Universidad de Antioquia. Corro electrónico: victor.molina@udea.edu.co. 


\section{INTRODUCCIÓN}

Se es libre cuando uno tiene tiempo libre para hacer con su vida lo que se le encanta, que es para cada cual su motivación... Para unos puede ser pescar; para otros, jugar al fútbol; para otros, estar durmiendo debajo de un árbol... José "Pepe" Mujica

Un tema de mayor relevancia hoy en día lo constituye la interculturalidad. La discusión alrededor de la diversidad cultural como condición determinante de la convivencia entre las personas y los pueblos, amerita problematizaciones en cuanto un rasgo identificador de la sociedad moderna lo representa, sin dudas, la actual tendencia homogeneizadora, muy útil al sistema político/económico imperante basado en la diferenciación y la desigualdad social (Díez, 2004).

A partir de esta tendencia, lo que se constata globalmente es un gravísimo problema de interculturalidad, donde la cultura - como espacio de reproducción social y organización de las diferencias - es negada. Se afirma, entonces, que en las actuales condiciones del sistema mundial capitalista de Occidente no hay una legítima globalización, ya que lo que se tiene es la globalización exitosa de un localismo dado, es decir, no hay una condición global para las raíces locales.

En el campo de la educación, en especial la de los territorios indígenas, esta tendencia adquiere presencia bajo la forma de imposición de discursos, prácticas y contenidos de aprendizaje ajenos a las comunidades con fuerte impacto en sus formas de vida (Bolaños y Tattay, 2013).

Para algunos actores educativos de la comunidad indígena, la interculturalidad no es aún un proyecto realizable en Colombia, pues al Estado le cuesta asumirse en una posición de reconocimiento de la diversidad y de posibilidad de lo otro. Para ellos, la ausencia de un proyecto que reconozca la interetnicidad es lo que ha propiciado el enfrentamiento y el exterminio del otro, del diferente; que no tiene por qué ser idéntico a nadie. Muchas de las confrontaciones tienen como actor principal al Estado, dado su carácter etnocéntrico, de intolerancia y de desconocimiento de lo otro, pues como expresión oficial de poder y de la clase mestiza, niega la esencia diferente de los colectivos indígenas.

Estas preocupaciones por la diversidad cultural dieron origen a la investigación "Cosmovisión de la unidad: existencia equilibrada, juego/producción y educación en la comunidad indígena nasa de Caldono, Colombia", la cual tuvo como objetivo principal identificar los rasgos configurativos de la cultura del ocio en el resguardo indígena de San Lorenzo de Caldono y su relación con la cultura del desarrollo sostenible.

Algunas categorías importantes para la investigación fueron: interculturalidad, juego/ocio, tiempo y escolaridad.

La interculturalidad se trabajó como una opción por la existencia de diversos mundos, como posibilidad de construir una sociedad a partir de una ética, una política y una economía de la descolonización de todas las formas de vida, esto es, como una práctica política ligada a la posición resistente de agrupamientos humanos sometidos a modelos organizativos impropios con los cuales se ha buscado hacerlos otros.

Desde la perspectiva de los pueblos indígenas, la interculturalidad representa el reconocimiento de las diversas racionalidades y cosmovisiones de los pueblos. Es una apuesta radical por la descolonización en los campos de lo económico, lo cultural, lo político y en las formas de concebir el mundo y la vida. Para la investigadora Catherine Walsh (2006), la interculturalidad representa la posibilidad que tienen los diferentes colectivos humanos de construir y vivir una epistemología otra, una práctica política otra, un poder estatal otro y unas relaciones sociales otras.

El juego/ocio se abordó como el conjunto de prácticas y manifestaciones de la comunidad relacionadas con la fiesta, las celebraciones, el descanso, los rituales, los juegos, las bebidas y la comida.

El tiempo se problematizó a partir de la comprensión que de él tienen los pueblos indígenas, como una coordenada existencial que adquiere presencia como un tiempo total, cíclico. Un tiempo no equiparado al dinero. Al decir del economista Juan Camilo Rodríguez (1988), cada forma de sociedad presenta particularidades en su configuración temporal. Es necesario entender que el tiempo se expresa como una forma de control social por la cual se pronuncian el poder y la dominación en una sociedad determinada. De allí que adquiere sentido para este escrito, la crítica de Fernando González (1929) sobre la forma como la sociedad actual relaciona el tiempo con el dinero.

La escolaridad fue entendida como los procesos de educación oficial estructurada y vigilada por el Estado colombiano. 


\section{METOdOLOGÍA}

La investigación se desarrolló a partir de la etnografía, entendida al modo de Clifford Geertz (2003) como descripción densa, que derivó, en la medida que avanzaba el trabajo, en una etnografía reflexiva con fundamento en los trabajos teóricos de Rossana Guber (2001), Maria Cecilia de Souza Minayo (2005) y en las elaboraciones de Pierre Bourdieu y Loïc Wacquant (2005), en especial de su sociología reflexiva.

La fase interpretativa se apoyó en las elaboraciones de Hans George Gadamer (1996) y María Cecilia de Souza Minayo (2005) prevalentemente. De esta autora se significó su perspectiva hermenéutica dialéctica, como ejercicio que destaca en la interpretación el carácter contextual y crítico de la acción hermenéutica, dando forma a la hermenéutica dialéctica; opción metodológica que asume posición a partir de la forma de ver el mundo del investigador, de allí su intertextualidad y complementación con la etnografía reflexiva de los autores antes citados.

La construcción del informe final se hizo a partir de las acciones realizadas en el territorio: estadía permanente en el resguardo (institución legal y sociopolítica de origen colonial integrada por una comunidad indígena con propiedad colectiva sobre su territorio y reconocida legalmente por el Estado colombiano), participación en una serie de actividades comunitarias importantes para realizar una aproximación a las tramas de sentido cultural de la comunidad como son: mingas, movilizaciones, rituales de refrescamiento, limpieza de varas de mando, carnavales, trueques, visitas a instituciones educativas, participación en proyectos productivos, capacitación en formas asociativas, actos de posesiones del Cabildo mayor, fiestas, celebraciones y eventos externos. El consentimiento fue obtenido por parte del Cabildo indígena del resguardo.

La estadía se registró en notas de campo (ANC), filmaciones, grabaciones, fotografías, acopio de documentos producidos por la misma comunidad, escritos varios y tesis de grado sobre asuntos de la colectividad nasa. Todos estos materiales fueron organizados a partir de los asuntos inicialmente problematizados. De este trabajo de campo se pasó a una segunda fase de reconstrucción del proceso vivido, donde a partir de un ejercicio de lectura en la sospecha se elaboró una intertextualidad de los tres planos de la investigación: descriptivo (la voz del otro), interpretativo (investigador) y de teoría social existente (textos de referencia del investigador).

Este ejercicio fue confrontado con la información obtenida de las entrevistas a personas clave del resguardo; evidencia empírica que es citada en la obra como entrevistas a actores internos (AEAI). Una construcción preliminar de este informe fue sometida a la mirada de actores internos y externos de la comunidad, lo que para el ejercicio se entendió, al decir del profesor Agustín Escolano (2000), como interpretación endógena y exógena, respectivamente. Para ello se buscaron interpretaciones de la información por parte de expertos de la misma comunidad en los campos de educación, gobernabilidad y liderazgo desde la máxima instancia de decisión, el Cabildo. En lo externo se recurrió a personas que tuvieran experiencia en la realización de investigaciones etnográficas, con fortaleza en el campo del ocio y la recreación. De esta manera, se accedió a la mirada y el sentido de tres personas con experiencia en el tema del ocio. Con esta doble interpretación se buscó profundizar en los criterios de verificabilidad y confiabilidad de la información elaborada.

La construcción del informe se hizo teniendo como referente las preguntas que inicialmente orientaron la investigación, sin restringirse literalmente a ellas.

\section{RESULTADOS Y DISCUSIÓN}

\section{Tiempo de juego/ocio y tiempo de producción}

En muchas situaciones de la vida de las comunidades indígenas resulta difícil establecer una diferencia palmaria entre las acciones que se podrían caracterizar como de juego, de ocio y las de la producción. Este planteamiento es mucho más explícito en las labores de la casa y de la tierra, donde al no identificarse una relación patrono/obrero, la disposición y uso del tiempo dependen de la autodirección de los sujetos involucrados en la actividad. Dicho de otra manera, al no generarse una relación de dependencia expresada bajo la forma de trabajar para alguien - para otro, para un patrono-, la labor realizada no se separa de las otras actividades de la cotidianidad; por ejemplo, la relación con la naturaleza y la ritualidad, lo que hace que tanto la acción como el cuerpo mismo de las personas no se asuman como un instrumento o medio para otra cosa, como bien podría ser para el trabajo y desde él, para la 
acumulación. Se respeta así la integralidad de la vivencia, donde en su ejecución no se experimenta una pérdida de la libertad como bien y como derecho, pues al no estar impregnada por el rendimiento, como coacción explícita o implícita, la libre determinación tiene cabida, y no se vive el trabajo como castigo o sufrimiento. Esta posibilidad dista mucho de la experiencia del trabajo dentro del contexto de lo industrial, en el cual la serialización de la acción impide una alteración de los ritmos impuestos - calculados - por la organización; si hay un rasgo distintivo y caracterizador de la vida en la empresa como forma por excelencia de la vida urbana e industrial es la continuidad, la no interrupción.

Es una apreciación diferente del tiempo, el cual es vivido como un continuo y no como una brega cotidiana, como sucede en las sociedades definidas por la relación patrono/obrero, caracterizadas casi siempre por la monotonía; percepción que queda claramente esbozada en las palabras de Fernando González (1929): “La vida del hombre sobre la tierra es brega y triste. Vivir es luchar con el tiempo, el cual nos arrastra, a pesar de resistirlo. ¡Qué horrible es, durante algunos días, vivir!" (p. 47). Para este autor, hoy se tiene una diferencia estructural con nuestros antepasados, pues mientras ellos vivían para la eternidad, nosotros vivimos para el tiempo, y somos - por esta misma condición- convertidos en sus esclavos. ¿Y por qué tenemos esto? Porque ya no pensamos en la eternidad:

[...] porque somos un manojo de segundos, lo supremo para nosotros es el dinero. También este se compone de centavos y con él se compra todo lo que se ha inventado para adornar el tiempo. Por eso, desde que Lutero descubrió que en Roma estaban vendiendo la eternidad, dejamos de creer en ella, pues es absolutamente evidente que todo lo venal es terreno. (González, 1929, p. 52)

Nuestra sociedad y los tiempos que nos ha tocado vivir se caracterizan por el afán de lucro, por una persecución sin cuartel del dinero; toda la vida se gasta en su consecución, de un modo tal, que determina la existencia de las personas reemplazando el interés que se tenía antes por la bondad del alma.

Este afán por el dinero nos ha hecho presa del tiempo, cada vez tenemos más necesidades de algo, lo que hace que vivamos en función de los minutos y los segundos. Por ello viajamos a velocidades cada vez mayores, para economizar el tiempo y poder funcionar adecuadamente en la sociedad apresurada. "Movimiento rápido a leguas por hora, a kilómetros por minuto [...]. Es necesario correr, acumular rápidamente, porque nos deja la vida. Este es el siglo del hombre que hace fortuna" (González, 1929, p. 54). Por el dinero se valora al hombre. Su ausencia o escasez lo hace un ser inferior, fracasado e incompetente; la pobreza es inferioridad, peligrosa y de mal gusto. La sociedad del desarrollo y de la acumulación precisa sujetos de acción, dinámicos, metódicos y atrevidos en contra la paciencia y la contención, virtudes de nuestros antepasados:

¡Honor al hombre de acción, al joven cazador, honorable, duro, superhombre, de egoencia desarrollada, cruel! ¡Honor al hombre seductor que ha metodizado todo en orden al dinero! El hombre de acción es hermoso. ¡Loor a nuestro hombre recto, de mirada firme, pletórico de ansias! (González, 1929, p. 55)

Para los indígenas nasas, en sentido contrario, en la labor están tanto el sujeto como la totalidad; cuando se trabaja se piensa y cuando se piensa se trabaja, por ello el trabajo de los médicos tradicionales no es inferior al de la persona que trabaja la tierra, por eso se valora más la actividad del trabajo - la labor - que el objeto - la cosa-. El trabajo comporta para la comunidad un bien y un principio fundamental desde el cual es posible la construcción de la casa, de la Gran Casa (territorio) y es necesario para pertenecer a ella.

Esta condición permite que la producción sea interrumpida para dar paso al juego. Un ejemplo es que los niños puedan estar con sus padres en todos los lugares, incluso los reservados para la labor. Para ilustrar este análisis, nos sirve el siguiente pasaje:

El carnicero arroja los desperdicios de la carne recién cortada a los perros que exasperados rodean el lugar. En esta actividad lo que en un momento inicial se aprecia como trabajo por parte del carnicero, de inmediato se transforma, e intempestivamente adquiere la condición de juego. El carnicero simula lanzar cerca o lejos las sobras a los hambrientos caninos y estos se desplazan o regresan. En esta actividad se desvanece la prevalencia de la acción central (el trabajo) que realiza el joven. La situación temporo-espacial se confunde entre el placer obtenido por la nueva realidad generada con los perros - observable en su rostro- y su responsabilidad en la preparación de la carne, que finalmente entregará al comprador - acción determinante de su labor-. (ANC, 204) 
Esta posibilidad es mucho más evidente en trabajos propios, como el cultivo; aquí la actividad que permite la supervivencia es susceptible de ser interrumpida en cualquier momento, pues a diferencia de Occidente su manejo del tiempo es mucho más flexible, es un tiempo de la vida, de la existencia, y es esto precisamente lo que hace viable que se pueda jugar, suspender y regresar de nuevo al trabajo. También porque el concepto y la representación del tiempo no existen tan fraccionados como para nosotros (los no indígenas).

Al decir de Lundgren (1992), nuestro concepto de tiempo deja de ser cíclico, producto de la industrialización que precisa de la secuencialización, lo que deriva en un disciplinamiento de lo temporal, donde el reloj hace su aparición, no tanto para medirlo, como para dividirlo. Lo que a su turno, secciona la relación entre el modo de producción y la naturaleza, dando lugar a la división del tiempo entre actividades de trabajo y de ocio. Un tiempo que hoy en día es pensado como una cantidad abstracta que puede dividirse en horas, minutos y segundos. Mientras que en las culturas indígenas el tiempo es mucho más cíclico, lo que permite una relación más estrecha con la naturaleza. Es el tiempo del día y de la noche.

Esta relación no fragmentaria del tiempo se evidencia en que los niños juegan a trabajar en pequeño y trabajan jugando. Aquí se confunden los sentidos caracterizadores de la acción. En un mismo momento, la acción puede representar juego para unos y trabajo para otros (resolución de necesidades de existencia). La confusión de sentidos adquiere también la forma colaborativa cuando el niño juega y el padre trabaja, y más claro aún, cuando estas acciones individuales se interrumpen por momentos y ambos trabajan o ambos juegan. Este cambio de roles es posible gracias a que no hay que rendir cuentas a un propietario. Los niños (los no escolarizados o en suspensión de esta ocupación) acompañan a sus padres al trabajo, esto es, al lugar donde se resuelve la supervivencia (ANC, 140).

O como cuando los niños ejecutan una acción y la interrumpen de forma abrupta, un juego donde no importa su fin, poniendo de manifiesto que la acción se hace porque sí, y con esto basta. La finalidad reside en ella, en su ejecución y nada más.

Los niños pequeños juegan con lo que se les atraviesa, en este caso es como si jugaran a la naturaleza. Mientras llueve, la niña de tres años recoge en un vaso plástico las goteras de agua que caen de las tejas y luego las vacía en el piso. Esto lo hace repetidamente y en silencio, no importa si se moja; al parecer ni percibe que su cuerpo está completamente empapado. Su rostro y su cabellera forman un bello cuadro con el rocío de la lluvia sobre su cuerpo. Luego se dedica a recoger hojas que arranca de un árbol del jardín de la casa de enseguida, y una a una las va sumando hasta formar un grupito que luego lleva al lavadero para echarles jabón como si las lavara-. Actividades que hace, y como si nada abandona. Es como si sus actividades nunca concluyeran, o mejor aún, no condujeran necesariamente a algo. Se hacen mientras se hacen, y al parecer con esto basta. En un corto lapso realiza dos actividades, las cuales abandona inesperadamente. (ANC, 212)

Esta forma de relación con el tiempo, y su uso, es resultado de las particularidades de cada cultura, estructurada a partir de eventos significativos de la comunidad, como lo señala Evans Pritchard (citado por Leff, 2000):

[...] el tiempo se estructura alrededor de eventos significativos, tanto sociales como económicos. De esta manera, cada cultura, define sus "temporalidades" a través de su cosmología y sus sistemas simbólicos (Pitt, 1985). El tiempo no es solo la medida de eventos externos (fenómenos geofísicos, ciclos ecológicos, procesos de degradación y regeneración de la naturaleza), sino el flujo de eventos internos significativos, la permanencia de "presencias" a través de la historia, la actualización de las identidades étnicas y "seres culturales". El tiempo libera a la naturaleza de-signada y fijada a visiones predeterminadas, abriendo los cauces para la construcción de una naturaleza resignificada por los sentidos de la identidad y la cultura. (p. 64)

Así entonces, muchas de las comunidades indígenas han edificado una forma específica de relación con el espacio y el tiempo en cada uno de sus territorios, que obedece más que a determinaciones naturales o culturales, a factores sociohistóricos. De tal suerte, el territorio se constituye en el corazón sobre el cual se tejen el pensamiento y la vida de los indígenas nasas; es el escenario donde se hace posible la integralidad, la unidad de los mundos cósmico, terrestre y subterráneo (Molina, 2009).

La forma en que la comunidad configura su ordenamiento territorial apunta indiscutiblemente al espacio, pues - como para cualquier colectivo- este es una construcción social nacida del tipo de relaciones sociales y de producción de una sociedad en particular. Esta clari- 
dad es importante al momento de entender la diferencia entre organización espacial y ordenamiento territorial, conceptos similares que conservan una diferencia sustantiva, pues lo espacial se refiere a la dimensión geográfica, en tanto el ordenamiento territorial alude a la actividad política y jurídica. Las decisiones en ambos campos deben ser analizadas desde una perspectiva temporal, pues son procesos activos y en permanente transformación, acordes con las tensiones tanto internas como externas. Dichas decisiones, en su gran mayoría, están atravesadas por aspectos espacio-temporales, ambientales, étnico-culturales, económicos y políticoideológicos (Fajardo y Gamboa, 1998).

\section{EL CRONOSISTEMA ESCOLAR}

La posibilidad de relación diversa con el tiempo y el espacio, anteriormente señalada, es en muchos casos, negada en la escuela (oficial) al establecer y delimitar los tiempos de manera acorde con un cronosistema que regula los lapsos del trabajo/estudio y el descanso. Esta segmentación es una de las tareas prioritarias de la escolarización, por la cual se busca alejar a los pequeños de los ciclos naturales e incorporarlos en su dimensión abstracta (Lundgren, 1992), donde el timbre y otros dispositivos demarcan las fronteras entre lo que se debe y no se debe hacer, e introducen el horario como organizador estructurante de la vida y la cotidianidad escolar. Esta relación temporal irrumpe en la tradición de los menores - pues su vida colectiva transita y se desenvuelve en un tiempo social global- y vulnera sus juegos al territorio, a la minga, a la revolcada, e impide igualmente prácticas tan sencillas como lanzar piedras y tierra a su modo. Para los menores no existe diferencia entre los horarios del trabajo y del descanso o del juego, ellos simplemente crean la ocasión para divertirse con los demás o lo demás.

Ya en el recinto delimitado de la escuela, sólo es posible jugar en el espacio temporal y espacial permitido por la autoridad y la arquitectura escolar. Exclusivamente se puede jugar en el descanso, en las clases de educación física y deportes - donde prevalece el modelo deportivista- y en los lugares asignados para ello, como los deslizaderos - los barrialeros para los pequeños-, en los que los niños en fila, uno a uno, trepan las llantas dispuestas en el árbol para luego rodarse.
Fotografía 1. Escenarios deportivos escolares.

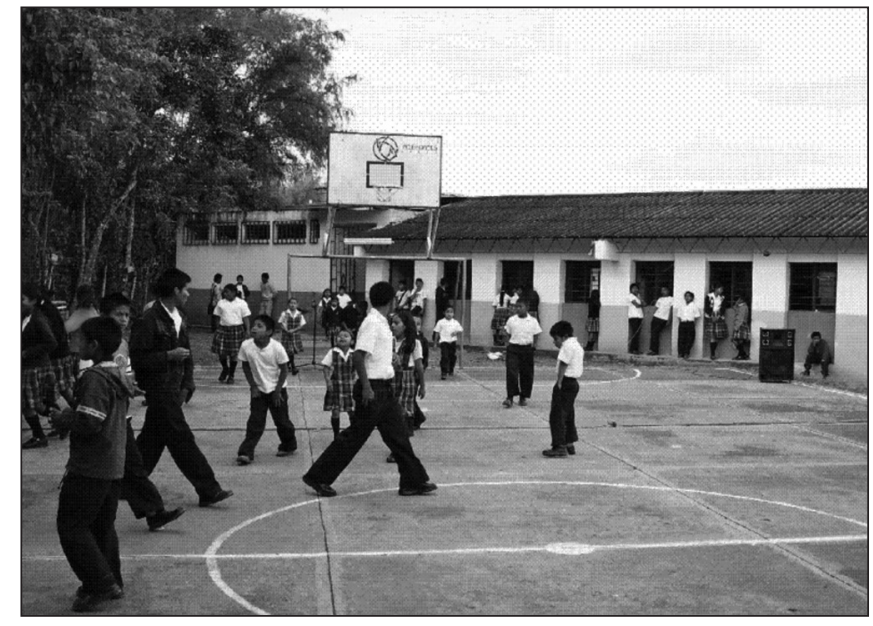

Fuente: autor

En otros sitios de la estructura escolar los más grandecitos juegan a La lleva (actividad que inicia cuando un niño emprende la persecución de los demás compañeritos y al tocarlos los convierte en los perseguidores, y así sucesivamente). El columpio es el otro dispositivo de mayor utilidad para los niños en las horas de descanso. Al salir de la escuela, es muy común verlos en el parque jugando a las bolas, a las canicas; sin importar el tiempo. Como se puede apreciar, la escuela cuadricula y encierra, además del pensamiento y el cuerpo, al juego mismo. (ANC, 300)

Desde otra cultura, pero alrededor de la misma valoración del escenario educativo como encierro, usamos la cita de los wiwas, para quienes:

La escuela formal como lugar encerrado en cuatro paredes llegó al territorio Wiwa desde inicios de este siglo; primero a La Sierrita y luego a Abingüe. Desde entonces entró en crisis la educación Wiwa. Urruama, el pensamiento, no se puede encerrar en una cárcel, hay que dejarlo que ande por todos lados. (Fajardo y Gamboa, 1998, p. 155)

La forma del emplazamiento físico del plantel reproduce la figura de la cuadrícula citadina; organización espacial que constriñe a los niños a pensar de una forma, a jugar a lo permitido, a los espacios diseñados por los planificadores urbano-escolares, para quienes pareciera no existir sino la cuadrícula, donde tanto los lugares para el descanso como las aulas para la educación, no pueden ser de otra forma: circulares, polimorfas, espiraladas, sin ventanas o sin paredes. Es decir, lo más cercano posible a los sitios de la comunidad, al territorio; a jugar al territorio (como un lugar ilimitado de juego y de posibilidades inmensas, ver más ampliamente en Molina, 
2012), lo que en otras palabras podría ser la actividad física en la naturaleza o los deportes en la naturaleza (Gomes, 2013).

Es un diseño espacial que cercena la posibilidad de jugar al territorio, a los árboles, a la naturaleza, de imaginar, de abrir los horizontes $a$ todo. Por el contrario, la escuela inicia a los infantes en el mundo de la vida y de la cultura acorde con los procesos de la socialización dominante.

Respecto a este tema, las investigaciones del maestro Agustín Escolano (2000) en el escenario de los tiempos y espacios de la escuela son claras cuando indican que "tomar posesión de la infancia, atribuirle tiempos y espacios, es ocupar sus disponibilidades, otorgar un determinado orden de cultura y sociabilidad a su espontaneidad" (s.p.). Para Peter McLaren (1994), por su parte, la forma como la cultura se inscribe en el cuerpo representa la penetración no discursiva de la carne, desde la localización física (disposición panóptica del espacio escolar) y desde el tatuaje cultural del cuerpo (código de vestimenta).

De esta forma el cuerpo/sujeto se convierte en el medio y en el fin de la formación subjetiva; la escuela inserta así al individuo tanto a un orden simbólico preconstituido como a su investidura afectiva. Como resultado, el sujeto se identifica y apropia de los símbolos suministrados por el discurso y el sujeto de la enunciación.

Asimismo, la escuela como institución no solo determina y define unos tiempos que se presentan como ajenos para los menores recién incorporados a sus aulas, sino que también modifica los tiempos de la familia y los tiempos sociales de la colectividad indígena que, por vías de la presión y la responsabilidad para con la educación de sus hijos, incorporan para sí los tiempos y espacios diseñados por el aparato disciplinador escolar. De allí que muchos mayores y médicos tradicionales (The Walas) responsabilicen a la escuela de la pérdida de la lengua propia y de muchas de las prácticas ancestrales que los caracterizan como pueblo, al propiciar una especie de invasión cultural donde lo externo se muestra como superior (Bustamante, 2008) y exhibe en cambio, a la cultura propia como inferior e innecesaria.

[...] se presenta un enfrentamiento entre la educación oficial y la educación propia, o educación como legado, es decir, aquella que se transmite por los mayores, los anteriores, a las nuevas generaciones, y casi siempre se da en la lengua propia, el Nasa Yuwe. (AEAI, p. 68).

Hay que tener presente que históricamente la educación de los nasas ha estado ligada a la familia, donde los niños aprenden las pautas de la colectividad, la relación con el territorio, los saberes ancestrales, los valores comunitarios y los conocimientos acumulados transmitidos de generación en generación por la tradición oral.

El pequeño en la escuela aprende así a diferenciar la existencia de unos espacios, como son los del trabajo, del estudio y del juego. Pero, sobre todo, aprende a manejar los repartos del tiempo, como lo hace la sociedad blanca mayoritaria. Un tiempo que como condición de existencia debe ser organizado según las pautas trazadas por el cronosistema civilizatorio. De esta forma, la apropiación de las estructuras de lo temporal (Escolano, 2000) se ha convertido en uno de los principales cometidos de la escuela como institución socializadora.

El concepto lineal del tiempo es, por tanto, el resultado de una construcción social que en muchos de los casos se exhibe como un fenómeno natural. Tiempo que la sociedad ha catalogado — según los periodos vividos por las personas- como edades, a las cuales les corresponde un determinado tipo de comportamiento socialmente establecido, lo que permite clasificar a los seres en normales y anormales, según sea el caso o la conducta.

Dicho manejo del tiempo - como condición estructurante de la existencia de los seres humanos - se expresa manipulado por la imposición y heterodirección en las formas de uso y apropiación por parte de las instituciones que, como la escolar, definen los tiempos y su duración; es el caso de los periodos para las vacaciones, el descanso, la fiesta, la religión y la política, entre otros (Escolano, 2000). Lo que hace explícita una relación de poder, en cuanto determinación de la duración, de lo que es y no es permitido, y del correspondiente sistema de sanciones a que da lugar su falta. De esta manera, el cronosistema escolar, además de favorecer la interiorización de la organización del tiempo, propicia la autorregulación de los comportamientos de la infancia. Los horarios institucionales son algo más que una organización formal de la educación, y en cambio representan un dispositivo eficaz de control, poder e influencia (Da Silva Mello et al., 2012). Precisamente, 
la disciplina escolar encarna un mecanismo de control, determinado por instancias de poder de un nivel mayor, en muchos casos no visible.

Este control del tiempo en las instituciones escolares de los territorios indígenas debe ser también objeto de reflexión y problematización desde la perspectiva intercultural, que reconozca el carácter sociohistórico de las prácticas y los procesos sociales (Quilaqueo et al., 2011). Implica esto, tener presentes las formaciones, estructuras, resistencias, la desigualdad social, las luchas y las movilizaciones por el cambio. La interculturalidad representa pues un asunto de tipo político, económico, social y epistemológico que expresa los conflictos de poder entre facciones sociales diferenciadas, subalternizadas y en resistencia. De allí que su implementación demanda cambios estructurales que trasciendan el reconocimiento de las particularidades étnicas y propicien, en cambio, modificaciones en el tipo de relaciones sociales imperantes (Sierra, 2004); esto es, la interculturalidad como una práctica política de resistencia al actual modelo de asimilación cultural dominante, que posibilite la emergencia de nuevas identidades para confrontar el proyecto de hegemonía homogénea impulsado por el orden global vigente.

Las palabras de Enrique Leff (2000) son estimulantes y esperanzadoras:

Hoy, la historia se forja por la re-emergencia del ser. Historias acalladas, que parecían haber perdido su memoria y sus tradiciones, se están resignificando por nuevos propósitos, orientando una política del ser y del tiempo, y reconstruyendo eventos históricos pasados a través de la actualización de significados oprimidos. (p. 66)

Para sintetizar, es preciso agregar con José Rozo Gauta, que toda sociedad por antigua que sea, tiene ideas y representaciones del tiempo y del espacio como nociones aprehendidas en la práctica del diario trabajar y transformar. Estas nociones se asientan en la lengua y en el discurso social, las cuales se nos presentan bajo la modalidad de pensamiento mágico-religioso. Por eso las nociones y representaciones espacio-temporales tienen que ver con el orden general de la sociedad, son coetáneas a los logros de las prácticas utilitarias y especulativas, y conforman una totalidad de pensamiento con los fragmentos aprehendidos /nominados del mundo (Rozo, 1997).

\section{CONSIDERACIONES FINALES}

La vida en los territorios indígenas, al igual que la relación con el tiempo y sus contenidos, son diferentes a la forma en que estos son experimentados por la población blanca, musxka. Es una vida caracterizada, aún, por la economía de la supervivencia y la reciprocidad, que permite una forma de relación con el territorio no tan expoliativa como la que genera la sociedad occidental, ya que el potencial mítico sacraliza, da sentido a la relación entre los seres humanos y el entorno natural.

El tiempo como coordenada existencial adquiere la forma de un tiempo total, cíclico, más integral que el nuestro. De allí que sea difícil, en algunas situaciones de los pueblos indígenas, como acontece con la comunidad nasa, establecer claramente una diferencia entre los tiempos de la producción y los del juego/ocio.

Como se ha señalado, en esta comunidad se presenta una relación no fragmentaria del tiempo, donde los niños juegan a trabajar y trabajan jugando. Esto es, que un mismo momento la acción puede representar juego para unos y trabajo para otros, generándose así una confusión de sentidos respecto a la actividad. Los nasas han construido, de esa manera, una forma de relación con el espacio y con el tiempo en sus territorios, resultado de factores sociohistóricos y culturales.

La segmentación, el reparto del tiempo es más un producto de la articulación a las dinámicas externas, expresada como una de las tareas prioritarias de la escolarización, por la cual se busca introducir a los estudiantes en la dimensión abstracta del tiempo.

Esta organización de lo temporal trasciende el plano de la institución educativa y llega a modificar los tiempos mismos de la familia y de la colectividad, pues la responsabilidad con la educación de los hijos hace que se incorporen los tiempos del aparato disciplinador escolar.

Así, en las instituciones educativas se experimenta un choque entre los tiempos de la cotidianidad de los menores y la organización horaria de las escuelas, lo mismo que de los espacios, lo que incide en el tipo de prácticas y aprendizajes, en este caso relacionados con el juego/ocio. Por consiguiente, tanto los profesores como la misma institución, son presentados socialmente como representantes de una cultura supuestamente superior que debe renovar la cultura, los ritmos, la lengua y las prácticas de los estudiantes. 


\section{REFERENCIAS BIBLIOGRÁFICAS}

Bolaños, G. y Tattay, L. (2013). La educación propia, una realidad oculta de resistencia educativa y cultural de los pueblos. En: Entretejidos de la educación popular en Colombia (pp. 65-80). Bogotá: Ediciones Desde Abajo, Consejo de Educación Popular de América Latina y el Caribe (CEAAL) y de Solidaridad Socialista (Bélgica)

Bourdieu, P. y Wacquant, L. (2005). Una invitación a la sociología reflexiva. Buenos Aires: Siglo xxI Editores.

Bustamante, L. (2008). Escritura y rendimiento académico del estudiantado Nasa de la Universidad de Antioquia. Medellín: Colección Asoprudea.

Da Silva Mello, A.; Sarmiento, K.; Dos Santos, W.; Rodrigues Da Costa, F. y Votre, S. (2012). Representações sociais sobre a Educação Física na Educação Infantil. Revista Brasileira de Ciências do Esporte 23(3), 443-455.

De Souza, M. (2006). O desafio do conhecimento. Pesquisa cualitativa em saude. São Paulo: Aderaldo \& Rothschild Editores.

Díez, M. (2004). Reflexiones en torno a la interculturalidad. Cuadernos de Antropología Social, 19, 191-213.

Escolano, A. (2000). Tiempos y espacios para la escuela. Ensayos históricos. Madrid: Editorial Biblioteca Nueva.

Escolano, A. (2006). Métodos de investigación. Seminario de investigación doctoral, trabajos de investigación tutelados. Valladolid: Universidad de Valladolid.

Fajardo, L. y Gamboa, J. (1998). Multiculturalismo y derechos humanos: una perspectiva desde el pueblo indígena Wiwa de la Sierra Nevada de Santa Marta. Bogotá: ESAP.

Gadamer, H. (1996). Verdad y método I. Salamanca: Ediciones Sígueme.

Geertz, C. (2003). La interpretación de las culturas. Barcelona: Editorial Gedisa.

Gomes de Asis, G. (2013). Esportes na natureza e atividades de aventura: uma terminologia aporética. Revista Brasileira de Ciências do Esporte 35(3), 687-700.

González, F. (1929). Viaje a pie. Medellín: Bolsilibros Bedout.

Guber, R. (2001). La etnografía. Método, campo y reflexividad. Bogotá: Grupo Editorial Norma.

Leff, E. (2000). Espacio, lugar y tiempo: la reapropiación social de la naturaleza y la construcción local de la racionalidad ambiental. Desenvolvimento e Meio Ambiente 1(1), 57-69.
Lundgren, U.P. (1992). Teoría del curriculum y escolarización. Madrid: Ediciones Morata.

McLaren, P. (1994). Pedagogía crítica, resistencia cultural y la producción del deseo. Buenos Aires: Aique Grupo Editor.

Molina, V. (2009). Cosmovisión de la unidad. Existencia equilibrada, juego/producción, educación y resistencia en la comunidad indígena Nasa de Caldono, Cauca. Tesis doctoral. Valladolid: Universidad de Valladolid.

Molina, V. (2012). De juegos y de territorios. Comprensiones otras del ocio en sociedades de la periferia. Revista Educación Física y Deporte 31(2), 1011-1017.

Quilaqueo, D.; Quintriqueo, S. y San Martín, D. (2011). Contenido de aprendizajes educativos mapuches para el marco conceptual de un currículum escolar intercultural. Estudios Pedagógicos 37(2), 233-248.

Rodríguez, J. (1988). Tiempo y ocio. Bogotá: Universidad Externado de Colombia.

Rozo, J. (1997). Espacio y tiempo entre los muiscas. Bogotá: Editorial el Búho.

Sierra, Z., (2004). Voces indígenas universitarias. Expectativas, vivencias y sueños. Medellín: Grupo Diverser Universidad de Antioquia.

Walsh, C. (2006). Interculturalidad y colonialidad del poder. Un pensamiento y posicionamiento otro desde la diferencia colonial. En: C. Walsh; A. García y W. Mignolo. Interculturalidad, descolonización del Estado y del conocimiento (pp. 21-70). Buenos Aires: Ediciones del Signo. 